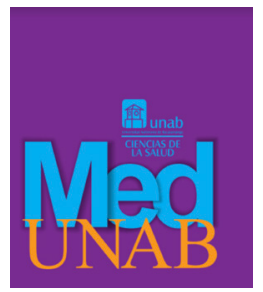

REVISTA DE LA FACULTAD

DE CIENCIAS DE LA SALUD

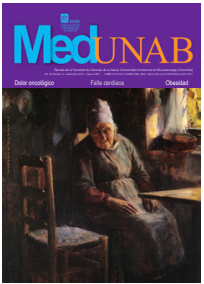

Editorial

\title{
Qualitative research: interactions and experiences
}

Investigación cualitativa: interacciones y experiencias

Pesquisa qualitativa: interações e experiências

Qualitative research depends on the views of the participants. The main purpose of qualitative research is to understand and interpret social interactions. Often the researcher wishes to explore a perplexing problem, providing a detailed understanding of a central phenomenon and seeks to appreciate the participants' experiences (1). The researcher collects information that is not in numerical form, which is typically descriptive data. Think of the word 'quality' - because we are taking a deep "quality" look at a phenomenon.

The researcher's role is unique in qualitative projects. Their biases may be known to participants in the study and participant characteristics are known to the researcher. This dynamic is different than data-driven, numerical investigations. The influence of the researcher's presence when collecting data and information must be recognized (2). Often this presence can affect the responses provided by participants in the study. This richness enhances the understanding of another individual's experiences.

Methods used in qualitative research collects data in the forms of words, images or objects.

Depending upon the approach, the researcher can utilize document reviews, participant observations, both individual and community, interviews, field notes, reflections by the researcher and participants, focus groups, and workshops (3).

There are six types of qualitative research utilized in various disciplines including sociology, anthropology as well as the heath fields of medicine, nursing, psychology, and social work $(4,5)$.

These models incorporate different data collection and analysis approaches (6). The following qualitative types of research are 1) ethnography, 2) grounded theory, 3) historical, 4) participant observation, 5) phenomenology, and 6) symbolic interactionism.

1. Ethnography involves the analysis or observation of a culture or social group's customs, rituals, and behaviors, and their understanding of disease and illness within that culture.

It requires methodical collection, detailed descriptions, and analysis of the information to help refine or develop cultural theories (4).

2. Grounded theory is often referred to as "theory building" because concepts, problems, and theories are continuously reevaluated. This 
approach is designed to discover what problems exist in a specific social environment and how these problems are resolved (4).

3. Historical research involves understanding and gathering information on the history of a topic, group, or culture. It allows the reader to systematically evaluate historical information via a detailed analysis of past occurrences (4).

4. Participant Observation is a foundational approach of involving the participants or subjects at the initial stages of discussion of a research project. These individuals of a select group, community or culture and interviewed in a variety of formats, attempting to identify and continuously adapt the types of gathered information, the analysis of the information and in a usual circular progression, adjust and re-structure the analysis and outcomes (5).

5. Phenomenology utilizes personal experiences to gain insight, empathy, or knowledge about a specific topic, situation, or "phenomenon." The investigator attempts to describe or understand a person's or group's perception, perspective, and understanding of a phenomenon and tries to answer the question, "What's it like to experience a given situation?" (4).

6. Symbolic interactionism focuses on patterns of communication, interpretation of that communication, and understanding how this communication forces adjustments between individuals who react to their interpretation of their world or social group. The narrative guides the reader to what has been proven to be beneficial or ineffective in certain situations in the past (4).

Analysis in all six types of qualitative studies seeks to explore, explain, or understand the collected information. Depending upon the selected method, the researcher utilizes narratives, a description of themes, or text analysis that states the larger meaning of findings (6).

Results are interpreted with a flexible, emerging structure and evaluative criteria.
The particular or specialized findings are limited in the generalizability and take a subjective biased approach (6). A final narrative report with contextual description and direct quotations from research participants provides an in-depth reflection of the experiences of persons in a select context.

Qualitative research provides the interwoven threads of a person's lived experiences, whether it is life as an outcast with AIDs, a person suffering from family violence, the joys of first time parenthood or the resilience of living as immigrants fleeing to uncertain lands.

\section{Sandra J. Cadena, Ph.D., APRN,} PMHCNS-BC, CNE

Fulbright Senior Specialist

Profesor Titular, Universidad El Bosque, Colombia, South America Senior Collaborating Faculty, Walden University

\section{References}

1. Denzin NK, Lincoln YS. The SAGE Handbook of Qualitative Research. 4th ed. Thousand Oaks, CA: Sage Publications; 2011.

2. Grove SK, Burns N. The Practice of Nursing Research: Appraisal, Synthesis, and Generation of Evidence. 6th ed. St. Louis, MO: Elsevier Saunders; 2008.

3. Hack L, Gwyer J. Evidence into Practice: Integrating Judgment, Values, and Research. Philadelphia, PA: F.A. Davis Co.; 2013:118-130.

4. Maxwell, JA. Qualitative Research Design: An Interactive Approach. 3rd ed. Sage Publications; 2013.

5. Munhall PL. Nursing Research: A Qualitative Perspective. 5th ed. Sudbury, MA: Jones \& Bartlett Learning; 2010.

6. Polit DF, Beck CT. Nursing Research: Generating and Assessing Evidence for Nursing Practice. 9th ed. Philadelphia, PA: Lippincott Williams \& Wilkins; 2011.

7. Wolcott HF. Writing Up Qualitative Research. 3rd ed. Newbury Park, CA: Sage Publications; 2008. 